\title{
Author Correction: Engineered reproductive tissues
}

Emma S. Gargus, Hunter B. Rogers, Kelly E. McKinnon, Maxwell E. Edmonds and Teresa K. Woodruff(D)

Correction to: Nature Biomedical Engineering https://doi.org/10.1038/s41551-020-0525-x, published online 6 April 2020.

In the version of this Review originally published, the authors mistakenly failed to include the following reference that has now been added as ref. 55:

55. Choi, J. K., Agarwal, P., Huang, H., Zhao, S. \& He, X. The crucial role of mechanical heterogeneity in regulating follicle development and ovulation with engineered ovarian microtissue. Biomaterials 35, 5122-5128 (2014).

This reference is cited in the sentence beginning "Follicles have been encapsulated..." and in the sentence beginning "(i) 3D structure of the.... All subsequent references have been renumbered accordingly.

Additionally, in the sentence beginning “(i) 3D structure of the..., 'alginate hydrogel' should have read 'collagen-alginate.' This error has been corrected.

Published online: 24 April 2020

https://doi.org/10.1038/s41551-020-0561-6

๑) Springer Nature Limited 2020 